\title{
ON THE GOODNESS OF FIT TEST FOR DISCRETELY OBSERVED SAMPLE FROM DIFFUSION PROCESSES: DIVERGENCE MEASURE APPROACH
}

\author{
SANGYEOL LEE
}

\begin{abstract}
In this paper, we study the divergence based goodness of fit test for partially observed sample from diffusion processes. In order to derive the limiting distribution of the test, we study the asymptotic behavior of the residual empirical process based on the observed sample. It is shown that the residual empirical process converges weakly to a Brownian bridge and the associated phi-divergence test has a chi-square limiting null distribution.
\end{abstract}

\section{Introduction}

The diffusion process has long been popular in analyzing random phenomena occurring in various fields such as finance, engineering, physical and medical sciences. As a basic reference, we refer to Karatzas and Shreve [5] and Shiryayev [20]. As the application of diffusion processes to real situations, especially to financial market, is versatile, much attention has been paid to statistical inference for diffusion processes and many sophisticated methods have been developed by researchers. For a general review, we refer to Prakasa Rao [18] and Kutoyants [7]. Past experience suggests that time series models such as general autoregressive conditional heteroscedastic (GARCH) models, which are the most popular in the financial time series context, are not well fitted to financial time series due to structural changes governed by the change of financial policies and social panic events. This phenomenon is frequently observed in most financial time series data with high volatility: see, for instance, Lee, Tokutsu, and Maekawa [13] who empirically verified, through the CUSUM test, that most stock prices of Nikei 225 suffer from parameter changes when the underlying model of the data is assumed to follow a $\operatorname{GARCH}(1,1)$ model. With regard to the parameter change test for time series models, we refer to Lee, $\mathrm{Ha}, \mathrm{Na}$, and $\mathrm{Na}$ [8], Lee and $\mathrm{Na}$ [10], Lee, Nishiyama, and Yoshida [11], and

Received January 8, 2009; Revised September 24, 2009.

2000 Mathematics Subject Classification. 60J60, $62 \mathrm{~F} 05$.

Key words and phrases. diffusion process, discretely observed sample, residual empirical process, weak convergence to a Brownian bridge, goodness of fit test, phi-divergence test. 
the articles cited in these papers. Since in general the stability of time series models is not guaranteed as mentioned above, instead of diffusion processes, researchers often consider using alternatives such as jump diffusion processes and Lévy processes: see Barndorff-Nielsen, Mikosch and Resnick [1], Shoutens [19] and Cont and Tankov [4]. Hence, in actual practice, it is very important to check whether time series follow diffusion or other processes.

In this paper, motivated by this viewpoint, we consider the goodness of fit test for diffusion processes. In particular, we concentrate on the phi-divergence test. The divergence measures are used as indices to similarity or dissimilarity between populations. They are also used either to measure mutual information concerning two r.v.'s and to construct goodness of fit tests. The most classic discrepancy is the Kullback-Leibler discrepancy while another important family of measures is the phi-divergence known as Csiszar's family of divergence measures of information on divergence methods: see Read and Cressie [17] and Pardo [16]. Recently, Lee and Karagrigoriou [9] rediscovered that there is a strong link between the phi-divergence test and empirical process, and verified that the asymptotic behavior of the phi-divergence test can be analyzed by studying that of the empirical process. In particular, they showed that the asymptotic results on the residual empirical process can be used for deriving the limiting distribution of the phi-divergence tests for autoregressive time series models.

Here, we employ the phi-divergence test constructed with the residuals obtained based on discretely observed sample from diffusion processes. We do this because if the data is generated from a pure diffusion process, the residuals obtained from the sampled observations should behave like normal r.v.'s. Then the whole testing procedure is reduced to the normality test for nearly i.i.d. observations. Since the phi-divergence test is strongly related to the residual empirical process, we study its asymptotic behavior in diffusion processes, which itself may be of an independent interest. In fact, the residual empirical process in time series models has been extensively studied by many authors. For instance, we refer to Lee and Wei [15], who deal with infinite order autoregressive models and unstable models, Boldin [2] and Lee and Taniguchi [12], who handled GARCH models, Lee and Wee [14], who consider the diffusion process with a constant dispersion component, and papers cited in those articles.

In Section 2, it is shown that under regularity conditions, the residual empirical process converges weakly to a Brownian bridge, and subsequently, by using the link between the residual empirical process and phi-divergence test, the latter has a chi-square limiting null distribution. Based on this result, one can easily perform a goodness of fit test in actual practice. Although we do not carry out an empirical study here, it is well known that the phi-divergence test performs adequately in other situations, and the same result is anticipated in our set-up. Finally, in Section 3, we provide the proof for the main theorem addressed in Section 2. 


\section{Main results}

Let us consider the stochastic differential equation

$$
d X_{t}=a\left(X_{t} ; \theta\right) d t+b\left(X_{t} ; \sigma\right) d W_{t}, \quad X_{0}=x_{0}, t \geq 0
$$

where $(\theta, \sigma)$ is a $p+q$-dimensional unknown parameter, $a, b$ are known real valued functions, and $W=\left\{W_{t} ; t \geq 0\right\}$ is a stochastic process that allows Equation (2.1) to have a unique strong solution. Further, we assume that

(A1) There exist constants $C, m>0$ such that for any $(\theta, \sigma)$ and $x, y$,

$$
\begin{aligned}
|a(x ; \theta)-a(y ; \theta)|+|b(x ; \sigma)-b(y ; \sigma)| & \leq C|x-y|, \\
\sup _{\theta^{\prime} \in N_{\theta}}\left\|\dot{a}\left(x ; \theta^{\prime}\right)\right\|+\sup _{\theta^{\prime} \in N_{\sigma}}\left\|\dot{b}\left(x ; \sigma^{\prime}\right)\right\| \leq C\left(1+|x|^{m}\right), & \leq \ddot{b} \sup _{\sigma^{\prime} \in N_{\sigma}}\left\|\ddot{b}\left(x ; \sigma^{\prime}\right)\right\| \leq C\left(1+|x|^{m}\right),
\end{aligned}
$$

where $\dot{a}=\partial a / \partial \theta, \ddot{a}=\partial a^{2} / \partial \theta^{2}, \dot{b}=\partial b / \partial \sigma, \ddot{a}=\partial a^{2} / \partial \sigma^{2}$, and $N_{\theta}$ and $N_{\sigma}$ are open neighborhoods of $\theta$ and $\sigma$.

$(\mathrm{A} 2) \inf _{x, \sigma} b(x, \sigma)>0$.

Here we consider the problem of testing the following hypotheses:

$$
H_{0}: W \text { is a standard Winer process vs. not } H_{0}
$$

via using the phi-divergence test introduced in Read and Cressie [17] and Pardo [16]. To task this, we study the asymptotic behavior of the residual empirical process constructed based on discreetly observed sample from the diffusion process in $(2.1)$, say, $X_{t_{i}}, t_{i}=i h_{n}, i=1, \ldots, n$, where $\left\{h_{n}\right\}$ is a sequence of positive real numbers such that $h_{n} \rightarrow 0$ and $n h_{n} \rightarrow \infty$.

In what follows, we assume

(A3) Under $H_{0}, \sup _{t} E\left|X_{t}\right|^{\gamma}<\infty$ for all $\gamma>0$.

Further, we assume

(A4) Under $H_{0}$, there exists an estimator $\left(\hat{\theta}_{n}, \hat{\sigma}_{n}\right)$ of $(\theta, \sigma)$ such that

$$
\left(n h_{n}\right)^{1 / 2}\left(\hat{\theta}_{n}-\theta\right)=O_{P}(1) \text { and } n^{1 / 2}\left(\hat{\sigma}_{n}-\sigma\right)=O_{P}(1),
$$

and

(A5) $n h_{n}^{2} \rightarrow 0$ and $\left(n h_{n}\right)^{1 / 2} / \log n \rightarrow \infty$ as $n \rightarrow \infty$.

Sufficient conditions for (A4) can be found in Kessler [6], which will be implicitly assumed without specification.

By using the Euler approximation, we can express

$$
X_{t_{i}}-X_{t_{i-1}} \simeq h_{n} a\left(X_{t_{i-1}} ; \theta\right)+b\left(X_{t_{i}} ; \sigma\right)\left(W_{t_{i}}-W_{t_{i-1}}\right) .
$$

In view of this, we define the residuals as

$$
\hat{\eta}_{n i}=\left\{X_{t_{i}}-X_{t_{i-1}}-h_{n} a\left(X_{t_{i-1}} ; \hat{\theta}_{n}\right)\right\} / b\left(X_{t_{i-1}} ; \hat{\sigma}_{n}\right) h_{n}^{1 / 2} .
$$


Since the phi-divergence test is strongly related to the residual empirical process, we consider the residual empirical process defined by

$$
\hat{Y}_{n}(x)=\frac{1}{\sqrt{n_{h}}} \sum_{i=1}^{n_{h}}\left\{I\left(\hat{\eta}_{n i} \leq x\right)-\Phi(x)\right\}, x \in \mathbb{R},
$$

where $n_{h}$ is the largest integer that does not exceed $n h_{n}$. Then we have the following result, the proof of which is provided in Section 3.

Theorem 2.1. Let

$$
\eta_{n i}=\frac{W_{t_{i}}-W_{t_{i-1}}}{\sqrt{h_{n}}} .
$$

Assume that (A1)-(A5) hold. Then, as $n \rightarrow \infty$,

$$
\hat{Y}_{n}(x)=\frac{1}{\sqrt{n_{h}}} \sum_{i=1}^{n_{h}}\left\{I\left(\eta_{n i} \leq x\right)-\Phi(x)\right\}+\xi_{n}(x),
$$

where $\sup _{x}\left|\xi_{n}(x)\right|=o_{P}(1)$. Thus, $Y_{n}\left(\Phi^{-1}(u)\right)$ converges weakly to a Brownian bridge $W^{o}(u)$.

Remark. Usually, the estimator of scale parameter affects the limiting distribution of the residual empirical process. In fact, this phenomenon can be found in GARCH processes (cf. Lee and Taniguchi [12]). In our case, however, the estimation effect disappears as seen in Theorem 2.1.

Lee and Wee [14] established the weak convergence of the residual empirical process to a Brownian bridge when the diffusion process has a constant dispersion: many well known stochastic differential equation (SDE) models in finance like the Black-Scholes model can be transformed to SDE models with a constant dispersion. In this case, one can even verify that their result holds for $n_{h}=n h^{\beta}$ with $0<\beta<1$ by virtue of (3.2) as long as $n h^{1+\beta} \rightarrow \infty$, although we would not provide a detailed proof for this. An advantage of using this $n_{h}$ is that the time horizon $n_{h} \cdot h$ diverges to $\infty$. This may not be possible for a general dispersion case.

Now, we introduce the phi-divergence test based on the residuals $\hat{\eta}_{n i}$. Let $\Lambda$ be a class of continuously twice differentiable real valued functions $\varphi$ defined on $\mathbb{R}^{+}$such that $\varphi(1)=0, \varphi^{\prime \prime}(1) \neq 0,0 \varphi(0 / 0)=0$ and $0 \varphi(p / 0)=\lim _{u \rightarrow \infty} \varphi(u) / u$. For $M \geq 2, \varphi \in \Lambda$, and the partition $A_{i}=\left(x_{i-1}, x_{i}\right]$, where $-\infty=x_{0}<x_{1}<$ $\cdots<x_{M}=\infty$ with $\Phi\left(x_{i}\right)=i / M$, the $\varphi$-divergence test based on the residuals is given by

$$
\hat{T}_{n}^{\varphi}=\frac{2 n}{\varphi^{\prime \prime}(1)} D_{\varphi}\left(\mathbf{p}, \hat{\mathbf{p}}_{n}\right)
$$

where $\mathbf{p}=\left(p_{1}, \ldots, p_{M}\right)^{\prime}$ and $\hat{\mathbf{p}}_{n}=\left(\hat{p}_{n 1}, \ldots, \hat{p}_{n M}\right)^{\prime}$ with $\hat{p}_{n i}=n^{-1} \sum_{j=1}^{n} I((i-$ $\left.1) / M<\Phi\left(\hat{\eta}_{n j}\right) \leq i / M\right)$, and $D_{\varphi}$ is a real valued function on $\mathbb{R}^{M} \times \mathbb{R}^{M}$, such that for all $M \times 1$ vectors $\mathbf{p}=\left(p_{1}, \ldots, p_{M}\right)^{\prime}$ and $\mathbf{q}=\left(q_{1}, \ldots, q_{M}\right)^{\prime}, D_{\varphi}(p, q)=$ $\sum_{i=1}^{M} p_{i} \varphi\left(q_{i} / p_{i}\right)$. In particular, when $\varphi(u)=\frac{u^{\lambda+1}-u}{\lambda(\lambda+1)}$ with $\lambda=-1 / 2,0,2 / 3,1$, 
the phi-divergence tests are called the Freeman-Tukey, likelihood ratio, CressieRead, and chi-square tests, respectively.

If we put for $s \in[0,1]$,

$$
\begin{aligned}
& \hat{\mathcal{Y}}_{n}(s)=\frac{1}{\sqrt{n_{h}}} \sum_{j=1}^{n_{h}}\left\{I\left(\Phi\left(\hat{\eta}_{n j}\right) \leq s\right)-s\right\}, \\
& \mathcal{Y}_{n}(s)=\frac{1}{\sqrt{n_{h}}} \sum_{j=1}^{n_{h}}\left\{I\left(\Phi\left(\eta_{n j}\right) \leq s\right)-s\right\},
\end{aligned}
$$

we can see that

$$
\hat{p}_{n i}=n_{h}^{-1 / 2}\left\{\hat{\mathcal{Y}}_{n}(i / M)-\hat{\mathcal{Y}}_{n}((i-1) / M)\right\}+1 / M
$$

Since $\hat{\mathcal{Y}}_{n}(s)=\hat{Y}_{n}\left(\Phi^{-1}(s)\right)$, by Theorem 2.1,

$$
\left(\hat{p}_{n i}-p_{i}\right)^{2}=n_{h}^{-1}\left\{\mathcal{Y}_{n}(i / M)-\mathcal{Y}_{n}((i-1) / M)+\zeta_{n i}\right\}^{2}=O_{P}\left(n_{h}^{-1}\right),
$$

where $\sup _{1 \leq i \leq M}\left|\zeta_{n i}\right|=o_{P}(1)$. Hence, in view of the arguments of Pardo [16, pp. 261-262], we can have

$$
\hat{T}_{n}^{\phi}=M \sum_{i=1}^{M}\left\{\mathcal{Y}_{n}(i / M)-\mathcal{Y}_{n}((i-1) / M)\right\}^{2}+o_{P}(1) .
$$

Subsequently, by Theorem 3.1 of Pardo [16], we have the following result.

Theorem 2.2. Under $H_{0}$ and the conditions in Theorem 2.1, as $n \rightarrow \infty$,

$$
\hat{T}_{n}^{\phi} \stackrel{d}{\rightarrow} \chi_{M-1}^{2},
$$

where $\chi_{M-1}^{2}$ denotes a r.v. with a chi-square distribution with $M-1$ degrees of freedom.

Given a significance level $\alpha \in(0,1)$, we reject $H_{0}$ if $T_{n}^{\phi} \geq \chi_{M-1}^{2}(\alpha)$ where $\chi_{M-1}^{2}(\alpha)$ is the number such that $P\left(\chi_{M-1}^{2} \geq \chi_{M-1}^{2}(\alpha)\right)=\alpha$.

\section{The proof of Theorem 2.1}

In this section, we prove Theorem 2.1. Put

$$
\begin{aligned}
\Delta_{n i} & =\int_{t_{i-1}}^{t_{i}}\left\{a\left(X_{s} ; \theta\right)-a\left(X_{t_{i-1}} ; \theta\right)\right\} d s+\int_{t_{i-1}}^{t_{i}}\left\{b\left(X_{s} ; \theta\right)-b\left(X_{t_{i-1}} ; \theta\right)\right\} d W_{s}, \\
d_{n i} & =a\left(X_{t_{i-1}} ; \hat{\theta}_{n}\right)-a\left(X_{t_{i-1}} ; \theta\right) .
\end{aligned}
$$

Note that $\Delta_{n i}$ can be viewed as a model bias in the regression model in (2.1). By using (A1), (A2), the arguments in the proof of Lemma 3.4.2 of Prakara Rao [18, p. 156], and the martingale moment inequality in Equation (3.27) of Karatzas and Shreve [5, p. 163], we can easily see that for each $k \geq 1$, there exists some $C_{k}>0$ depending only upon $k$ with

$$
E\left|X_{t}-X_{s}\right|^{2 k} \leq C_{k}|t-s|^{k} .
$$


Subsequently, by using Hölder's inequality, we can have

$$
E\left|\int_{t_{i-1}}^{t_{i}}\left\{a\left(X_{s} ; \theta\right)-a\left(X_{t_{i-1}} ; \theta\right)\right\} d s\right|^{2 k}=O\left(h_{n}^{3 k}\right) .
$$

Further, we can verify that

$$
E\left|\int_{t_{i-1}}^{t_{i}}\left\{b\left(X_{s} ; \sigma\right)-b\left(X_{t_{i-1}} ; \sigma\right)\right\} d W_{s}\right|^{2 k}=O\left(h_{n}^{2 k}\right) .
$$

Combining (3.2) and (3.3), we have

$$
E\left|\Delta_{n i}\right|^{2 k}=O\left(h_{n}^{2 k}\right) .
$$

We express that $Y_{n}(x)=I_{n}(x)+I I_{n}(x)+I I I_{n}(x)$, where

$$
\begin{aligned}
& I_{n}(x)=\frac{1}{\sqrt{n_{h}}} \sum_{i=1}^{n_{h}}\{I\left.\left(\eta_{n i} \leq x\right)-\Phi(x)\right\}, \\
& I I_{n}(x)=\frac{1}{\sqrt{n_{h}}} \sum_{i=1}^{n_{h}}\left\{\Phi\left(\frac{b\left(X_{t_{i-1}} ; \hat{\sigma}_{n}\right)}{b\left(X_{t_{i-1}} ; \sigma\right)} x-\frac{\Delta_{n i}}{b\left(X_{t_{i-1}} ; \sigma\right) h_{n}^{1 / 2}}-\frac{h_{n}^{1 / 2} d_{n i}}{b\left(X_{t_{i-1}} ; \sigma\right)}\right)-\Phi(x)\right\}, \\
& I I I_{n}(x)=\frac{1}{\sqrt{n_{h}}} \sum_{i=1}^{n_{h}}\left\{I\left(\eta_{n i} \leq \frac{b\left(X_{t_{i-1}} ; \hat{\sigma}_{n}\right)}{b\left(X_{t_{i-1}} ; \sigma\right)} x-\frac{\Delta_{n i}}{b\left(X_{t_{i-1}} ; \sigma\right) h_{n}^{1 / 2}}-\frac{h_{n}^{1 / 2} d_{n i}}{b\left(X_{t_{i-1}} ; \sigma\right)}\right)\right. \\
& \quad-\Phi\left(\frac{b\left(X_{t_{i-1}} ; \hat{\sigma}_{n}\right)}{b\left(X_{t_{i-1}} ; \sigma\right)} x-\frac{\Delta_{n i}}{b\left(X_{t_{i-1}} ; \sigma\right) h_{n}^{1 / 2}}-\frac{h_{n}^{1 / 2} d_{n i}}{b\left(X_{t_{i-1}} ; \sigma\right)}\right) \\
&\left.\quad+\Phi(x)-I\left(\eta_{n i} \leq x\right)\right\} .
\end{aligned}
$$

By Taylor's theorem, we can express $I I_{n}(x)=\sum_{i=1}^{4} I I_{n i}(x)$, where

$$
\begin{aligned}
& I I_{n 1}(x)=\frac{1}{\sqrt{n_{h}}} \sum_{i=1}^{n_{h}}\left(\frac{b\left(X_{t_{i-1}} ; \hat{\sigma}_{n}\right)}{b\left(X_{t_{i-1}} ; \sigma\right)}-1\right) x \phi(x), \\
& I I_{n 2}(x)=\frac{-1}{\sqrt{n_{h}}} \sum_{i=1}^{n_{h}} \frac{\Delta_{n i}}{b\left(X_{t_{i-1}} ; \sigma\right) \sqrt{h_{n}}} \phi(x), \\
& I I_{n 3}(x)=\frac{-1}{\sqrt{n_{h}}} \sum_{i=1}^{n_{h}} \frac{\sqrt{h_{n}}}{b\left(X_{t_{i-1}} ; \sigma\right)} d_{n i} \phi(x), \\
& I I_{n 4}(x)=\frac{1}{2 \sqrt{n_{h}}} \sum_{i=1}^{n_{h}}\left(\frac{\Delta_{n i}}{b\left(X_{t_{i-1}} ; \sigma\right) \sqrt{h_{n}}}+\frac{\sqrt{h_{n}}}{b\left(X_{t_{i-1}} ; \sigma\right)} d_{n i}\right)^{2} \phi^{\prime}\left(\xi_{n i}^{*}(x)\right),
\end{aligned}
$$

where $\xi_{n i}^{*}(x)$ is a number lying between $x$ and $\frac{b\left(X_{\left.t_{i-1} ; \hat{\sigma}_{n}\right)} b\left(X_{t_{i-1}} ; \sigma\right)\right.}{b}-\frac{\Delta_{n i}}{b\left(X_{t_{i-1}} ; \sigma\right) h_{n}^{1 / 2}}-$ $\frac{h_{n}^{1 / 2} d_{n i}}{b\left(X_{t_{i-1}} ; \sigma\right)}$. 
ON THE GOODNESS OF FIT TEST FOR DISCRETELY OBSERVED SAMPLE 1143

By (A1)-(A3) and the mean value theorem, we can easily see that

$$
\sup _{x}\left|I I_{n 1}(x)\right|=o_{P}(1) .
$$

On the other hand, by (3.4) we have

$$
E \sup _{x}\left|I I_{n 2}(x)\right|=o(1) .
$$

Now, by using Taylor's theorem, we express $d_{n i}=d_{n i}^{(1)}+d_{n i}^{(2)}$ with

$$
d_{n i}^{(1)}=\dot{a}\left(X_{t_{i-1}} ; \theta\right)\left(\hat{\theta_{n}}-\theta\right) \text { and } d_{n i}^{(2)}=\frac{1}{2}\left(\hat{\theta_{n}}-\theta\right)^{\prime} \ddot{a}\left(X_{t_{i-1}} ; \theta_{n i}^{*}\right)\left(\hat{\theta_{n}}-\theta\right),
$$

where $\theta_{n i}^{*}$ is an intermediate point between $\hat{\theta_{n}}$ and $\theta$. By (A1)-(A4), we can have

$$
\frac{1}{\sqrt{n_{h}}} \sum_{i=1}^{n_{h}} \frac{\sqrt{h_{n}}}{b\left(X_{t_{i-1}} ; \sigma\right)} d_{n i}^{(1)}=o_{P}(1)
$$

and

$$
\frac{1}{\sqrt{n_{h}}} \sum_{i=1}^{n_{h}} \frac{\sqrt{h_{n}}}{b\left(X_{t_{i-1}} ; \sigma\right)} d_{n i}^{(2)}=o_{P}(1)
$$

Hence,

$$
\sup _{x}\left|I I_{n 3}(x)\right|=o_{P}(1) .
$$

In a similar fashion, it can be seen that

$$
\sup _{x}\left|I I_{n 4}(x)\right|=o_{P}(1) .
$$

Hence, in view of (3.5)-(3.8), we have $\sup _{x}\left|I I_{n}(x)\right|=o_{P}(1)$.

Now it remains to verify that

$$
\sup _{x}\left|I I I_{n}(x)\right|=o_{P}(1) .
$$

Put

$$
w_{n}=\max _{1 \leq i \leq n_{h}}\left\{\frac{\sqrt{h}_{n}}{b\left(X_{t_{i-1}} ; \sigma\right)}\left|d_{n i}^{(2)}\right|+\frac{\left|\Delta_{n i}\right|}{b\left(X_{t_{i-1}} ; \sigma\right) \sqrt{h}_{n}}\right\} .
$$

Then, by (A1)-(A4), we can get

$$
n_{h}^{1 / 2} w_{n}=o_{P}\left(h_{n}^{1 / 2}\right) .
$$

Set

$$
e_{n i}=\frac{b\left(X_{t_{i-1}} ; \hat{\sigma}_{n}\right)}{b\left(X_{t_{i-1}} ; \sigma\right)}-1
$$

Observe that by the monotonicity of the indicator function,

$$
I\left(\eta_{n i} \leq x+\frac{\sqrt{h}_{n}}{b\left(X_{t_{i-1}} ; \sigma\right)} d_{n i}^{(1)}-\left[w_{n}+\max _{1 \leq i \leq n_{h}}\left(e_{n i} x\right)\right]\right)
$$




$$
\begin{aligned}
& \leq I\left(\eta_{n i} \leq \frac{b\left(X_{t_{i-1}} ; \hat{\sigma}_{n}\right)}{b\left(X_{t_{i-1}} ; \sigma\right)} x-\frac{\Delta_{n i}}{b\left(X_{t_{i-1}} ; \sigma\right) \sqrt{h}_{n}}+\frac{\sqrt{h}_{n}}{b\left(X_{t_{i-1}} ; \sigma\right)} d_{n i}\right) \\
& \leq I\left(\eta_{n i} \leq x+\frac{\sqrt{h}_{n}}{b\left(X_{t_{i-1}} ; \sigma\right)} d_{n i}^{(1)}+\left[w_{n}+\max _{1 \leq i \leq n_{h}}\left(e_{n i} x\right)\right]\right) .
\end{aligned}
$$

Further, by using (A1)-(A4), Taylor's theorem, (3.10), and the fact that

$$
n_{h}^{1 / 2} \max _{1 \leq i \leq n_{h}}\left|e_{n i}\right|=o_{P}(1),
$$

it can be readily checked that

$$
\begin{aligned}
\frac{1}{\sqrt{n}_{h}} \sum_{i=1}^{n_{h}} \mid \Phi\left(\frac{b\left(X_{t_{i-1}} ; \hat{\sigma}_{n}\right)}{b\left(X_{t_{i-1}} ; \sigma\right)} x-\frac{\Delta_{n i}}{b\left(X_{t_{i-1}} ; \sigma\right) \sqrt{h}_{n}}+\frac{\sqrt{h}_{n}}{b\left(X_{t_{i-1}} ; \sigma\right)} d_{n i}\right) \\
-\Phi\left(x+\frac{\sqrt{h}_{n}}{b\left(X_{t_{i-1}} ; \sigma\right)} d_{n i}^{(1)}+\left[w_{n}+\max _{1 \leq i \leq n_{h}}\left(e_{n i} x\right)\right]\right) \mid \\
+\frac{1}{\sqrt{n} h} \sum_{i=1}^{n_{h}} \mid \Phi\left(\frac{b\left(X_{t_{i-1}} ; \hat{\sigma}_{n}\right)}{b\left(X_{t_{i-1}} ; \sigma\right)} x-\frac{\Delta_{n i}}{b\left(X_{t_{i-1}} ; \sigma\right) \sqrt{h}_{n}}+\frac{\sqrt{h}_{n}}{b\left(X_{t_{i-1}} ; \sigma\right)} d_{n i}\right) \\
-\Phi\left(x+\frac{\sqrt{h}_{n}}{b\left(X_{t_{i-1}} ; \sigma\right)} d_{n i}^{(1)}-\left[w_{n}+\max _{1 \leq i \leq n_{h}}\left(e_{n i} x\right)\right]\right) \mid=o_{P}(1) .
\end{aligned}
$$

Subsequently, we have

$$
\begin{aligned}
& \sup _{x}\left|I I I_{n}(x)\right| \\
\leq & \sup _{x} \mid \frac{1}{\sqrt{n}} \sum_{i=1}^{n_{h}}\left\{I\left(\eta_{n i} \leq x+\frac{\sqrt{h}_{n}}{b\left(X_{t_{i-1}} ; \sigma\right)} d_{n i}^{(1)}+\left[w_{n}+\max _{1 \leq i \leq n_{h}}\left(e_{n i} x\right)\right]\right)\right. \\
& \left.-\Phi\left(x+\frac{\sqrt{h}_{n}}{b\left(X_{t_{i-1}} ; \sigma\right)} d_{n i}^{(1)}+\left[w_{n}+\max _{1 \leq i \leq n_{h}}\left(e_{n i} x\right)\right]\right)+\Phi(x)-I\left(\eta_{n i} \leq x\right)\right\} \mid \\
\leq & \sup _{x} \mid \frac{1}{\sqrt{n}} \sum_{i=1}^{n_{h}}\left\{I\left(\eta_{n i} \leq x+\frac{\sqrt{h}_{n}}{b\left(X_{t_{i-1}} ; \sigma\right)} d_{n i}^{(1)}-\left[w_{n}+\max _{1 \leq i \leq n_{h}}\left(e_{n i} x\right)\right]\right)\right. \\
& \left.-\Phi\left(x+\frac{\sqrt{h}_{n}}{b\left(X_{t_{i-1}} ; \sigma\right)} d_{n i}^{(1)}-\left[w_{n}+\max _{1 \leq i \leq n_{h}}\left(e_{n i} x\right)\right]\right)+\Phi(x)-I\left(\eta_{n i} \leq x\right)\right\} \mid \\
& +o_{P}(1),
\end{aligned}
$$

and therefore,

$$
\sup _{x}\left|I I I_{n}(x)\right| \leq \sup _{x}\left|I V_{n}(x)\right|+\sup _{x}\left|V_{n}(x)\right|+o_{P}(1),
$$

where

$$
I V_{n}(x)=\frac{1}{\sqrt{n}_{h}} \sum_{i=1}^{n_{h}}\left\{I\left(\eta_{n i} \leq x+\frac{\sqrt{h}_{n}}{b\left(X_{t_{i-1}} ; \sigma\right)} d_{n i}^{(1)}\right)\right.
$$


ON THE GOODNESS OF FIT TEST FOR DISCRETELY OBSERVED SAMPLE 1145

$$
\left.-\Phi\left(x+\frac{\sqrt{h}_{n}}{b\left(X_{t_{i-1}} ; \sigma\right)} d_{n i}^{(1)}\right)+\Phi(x)-I\left(\eta_{n i} \leq x\right)\right\}
$$

and

$$
\begin{aligned}
V_{n}(x)=\frac{1}{\sqrt{n}_{h}} \mid \sum_{i=1}^{n_{h}}\left\{I\left(\eta_{n i} \leq x+\left[w_{n}+\max _{1 \leq i \leq n_{h}}\left(e_{n i} x\right)\right]\right)\right. \\
\left.-\Phi\left(x+\left[w_{n}+\max _{1 \leq i \leq n_{h}}\left(e_{n i} x\right)\right]\right)+\Phi(x)-I\left(\eta_{n i} \leq x\right)\right\} \mid \\
+\frac{1}{\sqrt{n} h} \mid \sum_{i=1}^{n_{h}}\left\{I\left(\eta_{n i} \leq x-\left[w_{n}+\max _{1 \leq i \leq n_{h}}\left(e_{n i} x\right)\right]\right)\right. \\
\left.-\Phi\left(x-\left[w_{n}+\max _{1 \leq i \leq n_{h}}\left(e_{n i} x\right)\right]\right)+\Phi(x)-I\left(\eta_{n i} \leq x\right)\right\} \mid .
\end{aligned}
$$

Since

$$
\begin{aligned}
& \sup _{x}\left|V_{n}(x)\right| \\
\leq & 2 \sup _{|\Phi(x)-\Phi(y)| \leq b_{n}}\left|\frac{1}{\sqrt{n}_{h}} \sum_{i=1}^{n_{h}}\left\{I\left(\eta_{n i} \leq x\right)-\Phi(x)+\Phi(y)-I\left(\eta_{n i} \leq y\right)\right\}\right| \\
= & o_{P}(1)
\end{aligned}
$$

(cf. Billingsley $([3]))$, where $b_{n}=\sup _{x}\left|\Phi\left(x+\left[w_{n}+\max _{1 \leq i \leq n_{h}}\left(e_{n i} x\right)\right]\right)-\Phi(x)\right|=$ $o_{P}(1)$, it suffices to show that $\sup _{x}\left|V I_{n}(x)\right|=o_{P}(1)$.

Let $x_{j}$ be such that $-\infty=x_{0}<\cdots<x_{N_{n}}=\infty$ and $\Phi\left(x_{j}\right)=\frac{j}{N_{n}}, j=$ $0, \ldots, N_{n}$, where $N_{n}=n^{2}$. Since

$$
\max _{1 \leq j \leq N_{n}} \sup _{x_{j} \leq x \leq x_{j+1}}\left|\frac{1}{\sqrt{n_{h}}} \sum_{i=1}^{n_{h}}\left\{I\left(\eta_{n i} \leq x_{j}\right)-\Phi\left(x_{j}\right)+\Phi(x)-I\left(\eta_{n i} \leq x\right)\right\}\right|=o_{P}(1)
$$

in order to (3.9), it suffices to prove that

$$
\begin{aligned}
& \max _{1 \leq j \leq N_{n}} \mid \frac{1}{\sqrt{n}_{h}} \sum_{i=1}^{n_{h}}\left\{I\left(\eta_{n i} \leq x_{j}+\frac{\sqrt{h}_{n}}{b\left(X_{t_{i-1}} ; \sigma\right)} d_{n i}^{(1)}\right)\right. \\
& \left.-\Phi\left(x_{j}+\frac{\sqrt{h}_{n}}{b\left(X_{t_{i-1}} ; \sigma\right)} d_{n i}^{(1)}\right)+\Phi\left(x_{j}\right)-I\left(\eta_{n i} \leq x_{j}\right)\right\} \mid=o_{P}(1),
\end{aligned}
$$

which, however, can be verified by following essentially the same lines in Lee and Wei [15], Lee and Taniguchi [12], and Lee and Wee [14] and by using (A5) and Bernstein's inequality for martingales. For brevity, we complete the proof without detailing algebra.

Acknowledgements. This work was supported by grant No. R01-2006-00010545-0 from the Basic Research Program of the Korea Science \& Engineering Foundation. 


\section{References}

[1] O. E. Barndorff-Nielsen, T. Mikosch, and S. I. Resnick, Lévy Processes, Birkhauser Boston, Inc., Boston, MA, 2001.

[2] M. V. Boldin, On empirical processes in heteroscedastic time series and their use for hypothesis testing and estimation, Math. Methods Statist. 9 (2000), no. 1, 65-89.

[3] P. Billingsley, Convergence of Probability Measures, John Wiley \& Sons, Inc., New YorkLondon-Sydney, 1968.

[4] R. Cont and P. Tankov, Financial Modelling with Jump Processes, Chapman \& Hall/CRC, Boca Raton, FL, 2004.

5] I. Karatzas and S. E. Shreve, Brownian Motions and Stochastic Calculus, SpringerVerlag, New York, 1991.

[6] M. Kessler, Estimation of an ergodic diffusion from discrete observations, Scand. J. Statist. 24 (1997), no. 2, 211-229.

[7] Y. Kutoyants, Statistical Inference for Ergodic Diffusion Processes, Springer-Verlag London, Ltd., London, 2004.

[8] S. Lee, J. Ha, O. Na, and $\mathrm{S}$. Na, The cusum test for parameter change in time series models, Scand. J. Statist. 30 (2003), no. 4, 781-796.

[9] S. Lee and A. Karagrigoriou, On the $\phi$-divergence test for time series models, Submitted for publication (2008).

[10] S. Lee and O. Na, Test for parameter change in stochastic processes based on conditional least-squares estimator, J. Multivariate Anal. 93 (2005), no. 2, 375-393.

[11] S. Lee, Y. Nishiyama, and N. Yoshida, Test for parameter change in diffusion processes by cusum statistics based on one-step estimators, Ann. Inst. Statist. Math. 58 (2006), no. $2,211-222$

[12] S. Lee and M. Taniguchi, Asymptotic theory for ARCH-SM models: LAN and residual empirical processes, Statist. Sinica 15 (2005), no. 1, 215-234.

[13] S. Lee, Y. Tokutsu, and K. Maekawa, The cusum test for parameter change in regression models with ARCH errors, J. Japan Statist. Soc. 34 (2004), no. 2, 173-188.

[14] S. Lee and I. Wee, Residual empirical process for diffusion processes, J. Korean Math. Soc. 45 (2008), no. 3, 683-693.

[15] S. Lee and C. Z. Wei, On residual empirical processes of stochastic regression models with applications to time series, Ann. Statist. 27 (1999), no. 1, 237-261.

[16] L. Pardo, Statistical Inference Based on Divergence Measures, Chapman \& Hall/CRC, Boca Raton, FL, 2006.

[17] T. Read and N. Cressie, Goodness-of-Fit Statistics for Discrete Multivariate Data, Springer-Verlag, New York, 1988.

[18] B. L. S. Prakasa Rao, Statistical Inference for Diffusion Type Processes, Edward Arnold, London; Oxford University Press, New York, 1999.

[19] W. Schoutens, Lévy Processes in Finance, Wiley, New York, 2003.

[20] A. N. Shiryayev, Essentials of Stochastic Finance, World Scientific Publishing Co., Inc., River Edge, NJ, 1999

Department of Statistics

Seoul National University

SEOUL 151-742, KoreA

E-mail address: sylee@stats.snu.ac.kr 